\title{
Caractérisation zootechnique de génisses de races Holstein, Montbéliarde et Tarentaise*
}

\author{
P D'Hour 1, JB Coulon 1, M Petit 1, JP Garel 2 \\ 1 INRA-Theix, laboratoire d'adaptation des herbivores aux milieux, \\ 63122 Saint-Genès-Champanelle; \\ 2 INRA, domaine de la Borie, 15190 Marcenat, France
}

(Reçu le 7 mars 1994; accepté le 1er septembre 1994)

\begin{abstract}
Résumé - Le développement squelettique et pondéral et l'apparition de la puberté ont été décrits sur 72 génisses de 3 races laitières (Holstein, Montbéliarde et Tarentaise), élevées en milieu montagnard. À la naissance, les génisses Montbéliardes étaient plus lourdes $(P<0,05)$ que leurs homologues Holstein ( $41 \mathrm{vs} 38 \mathrm{~kg}$ ) et le sont restées jusqu'à l'âge de 4 mois. De l'âge de 12 à 24 mois, les génisses Holstein étaient significativement plus lourdes $(+20 \mathrm{~kg}$ environ) que les Montbéliardes $(P<0,01)$. Les génisses Tarentaises ont toujours été plus légères $(P<0,01)$ d'environ $30 \mathrm{~kg}$ que celles de race Montbéliarde. Pour les 32 animaux ayant vêlé 4 fois, les écarts entre races sont restés similaires jusqu'au $4 \mathrm{e}$ vêlage. Les courbes de croissance ont ensuite été ajustées par l'âge en utilisant le modèle descriptif de la croissance de Brody qui a donné des poids "adultes" non significativement différents pour les Holstein et les Montbéliardes (665 vs $641 \mathrm{~kg}$ ) et supérieurs $(P<0,05)$ à celui des Tarentaises $(618 \mathrm{~kg}$ ). Les rythmes de développement pondéral, estimé par ce modèle, des femelles Holstein et Montbéliardes $(0,0017)$ ont été semblables, et plus rapides $(P<0,05)$ que celui des Tarentaises $(0,00143)$. À tout âge, excepté à 6 mois, la plupart des mensurations étaient différentes $(P<0,05)$ entre les 3 races et les animaux se classaient dans l'ordre décroissant suivant : Holstein, Montbéliarde et Tarentaise, en particulier pour la hauteur au garrot, la profondeur de poitrine, ainsi que la largeur aux trochanters. Les génisses Holstein ont été pubères à un âge plus jeune $(305 \mathrm{j})(P<0,01)$ que les génisses Tarentaises (475 j) et Montbéliardes (504 j).
\end{abstract}

génisse laitière / race / croissance / puberté

Summary - Characterization of Holstein, Montbéliarde and Tarentaise heifers. Live weight and skeletal development, along with the onset of puberty, were described for 72 heifers belonging to 3 dairy breeds (Holstein, Montbéliarde, and Tarentaise), reared in a mountainous environment. At birth, the Montbéliarde heifers were heavier $(\mathrm{P}<0.05)$ than their Holstein counterparts $(41 \mathrm{vs} 38 \mathrm{~kg})$. Between the ages of 12 and 24 months, the Holstein heifers were around $20 \mathrm{~kg}(\mathrm{P}<0.01)$ heavier and the Tarentaise

\footnotetext{
* Travail réalisé dans le cadre du Programme de recherche-développement des Alpes du Nord
} 
around $30 \mathrm{~kg}(\mathrm{P}<0.01)$ lighter than the Montbéliarde. For the 32 animals having calved 4 times, these differences remained similar until the 4th calving. The growth curves were then adjusted according to age, by using a descriptive growth model (Brody), giving a mature live weight that was closely similar (NS) for Holstein and Montbeliarde (665 vs $641 \mathrm{~kg}$ ) and higher $(\mathrm{P}<0.05)$ than that of Tarentaise heifers $(618 \mathrm{~kg})$. The maturing rate, estimated with this model, of Holstein and Montbeliarde females (0.0017) was similar and faster $(\mathrm{P}<0.05)$ than that of the Trentaise (0.00143). At any given age, except 6 months, most of the measurements were different $(P<0.05)$ between the 3 breeds and the animals ranked in the following decreasing order: Holstein, Montbéliarde and Tarentaise; especially for wither-height and chest-depth as well as width between trochanters. The Holstein heifers reached puberty at a younger age (305 d) than Tarentaise (475 d) and Montbéliarde (504 d) heifers $(P<0.01)$.

dairy heifer / breed / growth / puberty

\section{INTRODUCTION}

Les performances de production et de reproduction des vaches laitières dépendent en partie des conditions d'élevage des génisses, et en particulier du développement et du poids qu'elles ont atteints à certaines périodes clés : il existe notamment un optimum de croissance pré- et péripubertaire, de poids à la mise à la reproduction et de poids au premier vêlage (Troccon et Petit, 1989). Les études descriptives sur le développement des génisses laitières ont en majorité été effectuées avec des races à haut potentiel laitier, principalement Holstein, même si quelques études ont concerné des races de potentiel plus modeste (Pie-Rouge Danoise et Suédoise, Frisonne Anglaise), produisant de 3000 à $4200 \mathrm{~kg}$ de lait en première lactation (Hansson et al, 1967; Foldager et al, 1978; Little et Kay, 1979).

Les objectifs de ce travail étaient de caractériser, dans un même milieu montagnard limitant sur le plan nutritionnel, la croissance et le développement squelettique de génisses de 3 races laitières utilisées en France dans certaines régions d'altitude : Tarentaise (potentiel laitier modéré), Montbéliarde (potentiel laitier plus élevé) et Holstein (laitière hautement spécialisée). La description a également porté sur l'apparition de la puberté. Ces observations ont été réalisées principalement de la naissance (de l'âge de 5 mois pour les Tarentaises) à 35 mois (avant le premier vêlage), mais des pesées effectuées au cours de la vie productive ont également été utilisées pour certaines analyses. Ce travail a été effectué sur le domaine INRA de Marcenat, situé dans le Massif central entre 1050 et $1150 \mathrm{~m}$ d'altitude sur le plateau humide et froid du Cézallier. La température moyenne annuelle n'y est que de $6,7^{\circ} \mathrm{C}$, et la saison de pâturage ne dure que 6 mois, de mi-mai à mi-novembre.

\section{MATÉRIEL ET MÉTHODES}

\section{Animaux et conduite d'élevage}

Les 72 génisses utilisées dans cette étude sont nées entre octobre 1986 et mars 1987. Les 22 génisses Holstein $(\mathrm{Ho})$ et les 17 Montbéliardes (Mo) sont issues du troupeau du domaine de Marcenat. Les 33 génisses Tarentaises ( $\mathrm{Ta}$ ), en provenance de différents élevages des départements de Savoie (27 génisses), Isère (2), HautesAlpes (2) et Haute-Loire (2), sont arrivées sur le domaine à l'âge moyen de 5 mois. Toutes les génisses étaient issues de 4 à 8 pères par race. favorablement indexés sur la production et la composition du lait

Les génisses ont toutes été conduites dans les mêmes conditions tout au long de l'étude. Elles ont hiverné en stabuiation entravée $(70 \%)$, ou en stabulation libre par race. Les génisses des 3 races étaient ensemble au pâturage. Dans 
ces conditions d'altitude, la courte durée de pâturage et la nature des fourrages hivernaux (foin de qualité moyenne : 0,62 à $0,65 \mathrm{UFL} / \mathrm{kg}$ de MS) ne permettaient pas d'envisager un vêlage précoce vers 2 ans pour l'ensemble des génisses. Nous avons donc choisi un premier vêlage à un âge plus tardif (33 à 36 mois), permettant de mieux grouper ces vêlages en fin d'automne et en tout début d'hiver, la même année. Les génisses ont été mises à la reproduction, début février 1989 , alors qu'elles étaient âgées de 24 à 27 mois. Après leur premier vêlage, les vaches ont été conduites toutes ensemble, que ce soit à l'étable (stabulation entravée) ou au pâturage.

Des traitements prophylactiques et antiparasitaires ont été réalisés régulièrement, au cours des saisons de pâturage (strongles, mouches), après la rentrée à l'étable (douves, varron et galles) et avant chaque mise à l'herbe (vaccin multivalent contre les entérotoxémies, le tétanos, le charbon et la gangrène gazeuse).

Pendant leurs 10 premières sem, les génisses Holstein et Montbéliardes ont reçu du lait de remplacement ( $60 \mathrm{~kg}$ d'aliment d'allaitement au total) et, à partir de l'âge de $4 \mathrm{sem}$, du foin et de l'aliment concentré en quantités croissantes, jusqu'à $1 \mathrm{~kg} / \mathrm{jour}$ de chacun des 2 aliments. Après leur sevrage, les génisses des 3 races ont reçu quotidiennement $2 \mathrm{~kg}$ de foin et $2 \mathrm{~kg}$ d'aliment concentré pour veaux d'élevage. Elles ont été mises à l'herbe tardivement, à la mi-juillet, à l'âge de 8 mois environ. Au cours de la première saison de pâturage (de 8 à 12 mois), toutes les génisses ont reçu un supplément de $1 \mathrm{~kg}$ de concentré par jour ( $2 \mathrm{~kg}$ au cours du dernier mois). Lors des 2 saisons de pâturage suivantes, aucune complémentation n'a été fournie. Elles ont pâturé au printemps (mi-mai) des prairies naturelles de fauche proches des étables $(1050 \mathrm{~m})$ pendant 2 sem environ, puis ont estivé ( $1100-1150 \mathrm{~m}$ ) durant environ 5 mois. La rentrée à l'étable (15-20 novembre) a suivi de près la descente d'estive. Les quantités d'herbe disponible ont généralement été faibles durant les 3 dernières sem de pâturage. Pendant les 2 hivernages, les génisses de 1 et 2 ans ont reçu à volonté des foins de différentes qualités (D'Hour et al, 1991). Pendant la période de stabulation hivernale, les vaches ont reçu une ration de base constituée de foin de prairie naturelle distribué à volonté $(0,70$ UFL $/ \mathrm{kg})$ et d'aliment concentré $(1,07 \mathrm{UFL} / \mathrm{kg})$. En période estivale, les vaches pâturaient des prairies naturelles proches de l'étable (Coulon et D'Hour, 1994).

\section{Mesures}

Les génisses et vaches étaient pesées tous les $14 \mathrm{j}$ depuis leur naissance (Ho et Mo) ou depuis leur achat $(\mathrm{Ta})$ jusqu'à leur premier vêlage. Une sem avant et 2 sem après la rentrée à l'étable et la sortie au pâturage, elles étaient pesées $2 \mathrm{j}$ successifs. Elles l'ont aussi été toutes les 2 à 3 sem durant la phase productive jusqu'à 56 mois ( 15 $\mathrm{Ho}, 13 \mathrm{Mo}$ et $20 \mathrm{Ta}$ ), et lors des troisième et quatrième $\mathrm{j}$ après les vêlages pour les 32 vaches restantes au quatrième vêlage $(8 \mathrm{Ho}, 10$ Mo et 14 Ta).

Les mensurations suivantes ont été réalisées au début et à la fin de chaque période hivernale (72 génisses), à partir de l'âge de 5 mois et jusqu'à 35 mois (avant le premier vêlage) : hauteur au garrot, tour et profondeur de poitrine, largeurs aux hanches et aux trochanters, longueur du corps (distance entre la pointe des ischions et celle de l'épaule).

L'âge à la puberté (apparition du premier cycle œstral) a été apprécié par les variations de la progestéronémie plasmatique (Thimonier, 1978) : 2 prélèvements sanguins à $10 \mathrm{j}$ d'intervalle ont été effectués mensuellement sur 60 génisses (15 Ho, $15 \mathrm{Mo}, 30 \mathrm{Ta}$ ). Les prélèvements ont débuté en juillet 1987 (soit à partir de 9 mois pour les génisses les plus âgées) et ont été poursuivis jusqu'à ce que toutes les génisses soient cyclées (août 1988). Les prélèvements ont été refaits et dosés en décembre et janvier 1989 afin de contrôler d'éventuels retours en anœstrus durant l'hivernage vers 2 ans.

\section{Analyses des données}

Le nombre d'animaux retenu a varié selon les objectifs des analyses. L'ensemble des génisses a été retenu pour caractériser la période d'élevage (de 5 à 35 mois). Au-delà du premier vêlage, n'ont été utilisées que les données correspondant aux vaches présentes. Les données des vaches réformées (malades, non gestantes ou vêlant tardivement) ont alors été éliminées.

Les gains de poids quotidiens moyens ont été calculés pour chaque période d'hivernage et de pâturage, entre les pesées effectuées 2 j successifs au début et à la fin de chacune de ces périodes. Les poids des 72 génisses aux âgestypes de $6,12,18,24$ et 30 mois (avant tout vêlage) ont été calculés par intrapolation. 
Les courbes individuelles de croissance pondérale ont ensuite été ajustées, en fonction de l'âge. Ce calcul a concerné 48 animaux (15 Ho, 13 Mo et $20 \mathrm{Ta}$ ) ayant réalisé au moins 2 lactations complètes, les autres ayant été réformés lors des 2 premières lactations. Un modèle de régression non linéaire (procédure NLIN ; SAS, 1987) a été utilisé sur l'ensemble des pesées réalisées de 5 mois jusqu'au milieu de la seconde lactation, correspondant à un âge moyen de 56 mois (95 à 125 pesées/génisse). Un tel ajustement a permis de limiter les fluctuations de poids vif qui pourraient résulter des variations de contenu digestif (lors des transitions alimentaires par exemple) ou de contenu utérin (autour du vêlage). Le modèle retenu a été celui de Brody (1945), qui reste utilisable lorsque les poids dans le jeune âge ne sont pas connus (cas des Ta), et dont les paramètres ont une signification biologique simple (Fitzugh, 1976 ; Perroto et al, 1992). Ce modèle est de la forme $P=P a-B \exp$ $(-k t)$ où $P$ est le poids vif (en $\mathrm{kg}$ ) de l'animal à un âge donné $t(\mathrm{j})$. L'asymptote $P$ a peut être interprétée comme le poids adulte, $B$ comme le gain de poids nécessaire du stade initial à l'âge adulte, et $k$ comme la vitesse de développement pondéral (vitesse de croissance rapportée au gain de poids restant à acquérir). Les variations de ces paramètres $P a, B$ et $k$ selon la race ont été étudiées par un modèle d'analyse de variance (procédure GLM; SAS, 1987).

À chaque période, les mensurations squelettiques ont été comparées entre races en les ajustant par l'âge par un modèle d'analyse de variance-covariance (procédure GLM ; SAS, 1987) tenant compte de la race (facteur fixe) et de l'âge (covariable) au moment de la mesure. Afin de prendre en compte le format des animaux, une deuxième analyse été réalisée sur le même modèle en y ajoutant en covariable la hauteur au garrot correspondante.

Enfin on a arbitrairement retenu, comme date d'apparition de la puberté, le jour de prélèvement sanguin où le taux de progestérone plasmatique était supérieur, pour la première fois, à $1,5 \mathrm{ng} / \mathrm{l}$ (Thimonier, 1978). Le poids à la puberté a été déterminé par intrapolation des 2 pesées encadrant ce jour.

\section{RÉSULTATS ET DISCUSSION}

Au total, $59 \%$ des génisses ont eu au moins une fois un trouble sanitaire. Les broncho- pneumonies infectieuses à l'étable entre 12 et 16 mois ont représenté l'essentiel (60\%) de ces troubles qui ont affecté de la même façon les génisses des 3 races. Huit génisses (Ho: 2, Mo: 2, Ta: 4) ont été réformées à la fin de leur troisième année, avant leur premier vêlage, en raison de difficultés de reproduction (génisses non gestantes ou vêlage attendu après le 15 février). Par la suite, outre les difficultés de reproduction, les réformes ont été décidées à la suite de troubles sanitaires (mammites en particulier).

\section{Évolution des poids et gains de poids}

Les génisses Holstein ont toujours été les plus lourdes et les Tarentaises les plus légères, quelle qu'ait été la période (tableau I) sauf dans le tout jeune âge. À ce moment, les génisses Montbéliardes étaient plus lourdes $(P<0,05)$ que les Holstein (41 vs $38 \mathrm{~kg}$ ). Cet écart de poids entre Holstein et Montbéliardes s'est inversé dès 6 mois (4 kg, NS) pour s'accroître la première année au cours de la saison de pâturage, passant à $21 \mathrm{~kg}$ à 1 an $(P<0,05)$, puis se stabilisant autour de $25 \mathrm{~kg}(P<0,05)$. À 30 mois, cet écart était encore de $19 \mathrm{~kg}$, quoique non significatif en raison notamment d'une plus grande dispersion des valeurs (tableau I). Les Tarentaises ont toujours été plus légères que les Montbéliardes, l'écart de poids variant de $29 \mathrm{~kg}$ à 6 mois à $57 \mathrm{~kg}$ à 30 mois $(P<0,01)$. Les croissances des génisses Holstein pendant le premier été ont été supérieures $(P<0,05)$ à celles des 2 autres races (tableau I). Par la suite, les génisses Holstein et Montbéliardes ont eu des croissances similaires, et toujours supérieures $(P<0,05)$ à celles des Tarentaises, excepté au cours du second été (vers 18 mois) où elles ont été semblables. Les écarts de poids constatés entre les races au cours de la période d'élevage se retrouvent durant la période productive, jusqu'au 
Tableau I. Moyennes ajustées (moindres carrés) des poids, des croissances et des poids à âges types de génisses laitières selon leur race.

\begin{tabular}{|c|c|c|c|c|}
\hline \multirow[t]{4}{*}{ 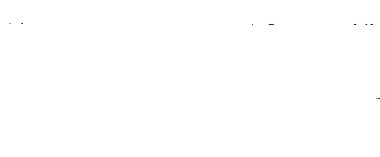 } & \multirow{2}{*}{\multicolumn{3}{|c|}{ Race }} & \multirow{3}{*}{ - } \\
\hline & & & & \\
\hline & \multirow{2}{*}{ Holstein } & - & - & \\
\hline & & Montbéliarde & Tarentaise & $E T R^{*}$ \\
\hline Effectif & 22 & 17 & 33 & \\
\hline Date de naissance moyenne & $23 / 11 / 86$ & $16 / 12 / 86$ & $1 / 12 / 86$ & \\
\hline \multicolumn{5}{|l|}{ Poids (kg) } \\
\hline Début 1 er été $(2 / 07)$ & $202^{a}$ & $189^{a b}$ & $178^{b}$ & 33 \\
\hline Début 1 er hiver (1/12) & $278^{a}$ & $248^{b}$ & $237 \mathrm{c}$ & 29 \\
\hline Début $2^{e}$ été $(3 / 05)$ & $361^{\alpha}$ & $331^{b \beta}$ & $312^{c} \beta$ & 33 \\
\hline Début $2^{e}$ hiver (24/11) & $454^{\mathrm{a} \alpha}$ & $430^{b a x}$ & $398^{c} \beta$ & 36 \\
\hline Début $3 e$ été $(26 / 04)$ & $509 a \alpha$ & $484^{b c x}$ & $440^{c} \beta$ & 37 \\
\hline Fin $3^{e}$ été $(7 / 10)$ & $590^{x}$ & $571^{x}$ & $507 \beta$ & 40 \\
\hline 1er vêlage $e^{\star \star}$ & $587(8)^{\alpha}$ & $579(10)^{\alpha}$ & $527(14)^{\beta}$ & 31 \\
\hline $2^{e}$ vêlage $^{\star \star}$ & $612(8)^{\alpha}$ & $591(10)^{\alpha}$ & $558(14)^{\beta}$ & 37 \\
\hline $3^{e}$ vêlage ${ }^{* \star}$ & $643(8)^{\alpha}$ & $631(10)^{\alpha}$ & $588(14)^{\beta}$ & 43 \\
\hline $4^{e}$ vêlage ${ }^{\star *}$ & $671(8)^{\alpha}$ & $648(10)^{\alpha}$ & $604(14)^{\beta}$ & 47 \\
\hline \multicolumn{5}{|l|}{ Croissance $(g / j)$} \\
\hline 1er été & $498^{a}$ & $389^{b}$ & $386^{\mathrm{b}}$ & 187 \\
\hline 1 er hiver & 539 & 539 & 484 & 109 \\
\hline $2^{e}$ été & $451^{a b}$ & $482^{a}$ & $419^{b}$ & 106 \\
\hline $2^{\mathrm{e}}$ hiver & $365^{a}$ & $352^{\mathrm{a}}$ & $277^{b}$ & 123 \\
\hline $3^{e}$ été & $493^{\alpha}$ & $532^{\alpha x}$ & $409^{\beta}$ & 112 \\
\hline \multicolumn{5}{|l|}{ Poids à âge type } \\
\hline Naissance & $38^{a}$ & $41^{b}$ & - & 5 \\
\hline $180 \mathrm{j}$ & $185^{a}$ & $181^{a}$ & $152^{b}$ & 19 \\
\hline $365 j$ & $308^{a \alpha}$ & $287^{b x} x$ & $259 c \beta$ & 29 \\
\hline $545 j$ & $382^{a \alpha}$ & $363^{b x}$ & $330^{\mathrm{c \beta}}$ & 27 \\
\hline $730 \mathrm{j}$ & $460^{a} \alpha$ & $439 \mathrm{bc} x$ & $399 \mathrm{c \beta}$ & 33 \\
\hline $910 \mathrm{j}$ & $538^{\alpha}$ & $520^{\alpha}$ & $463^{\beta}$ & 40 \\
\hline
\end{tabular}

\footnotetext{
* Écart type résiduel. ** Poids mesuré les $4^{e}$ et $5^{e}$ j après le vêlage (nombre de vaches ayant réalisé 4 vêlages) Entre races, des lettres différentes indiquent des différences significatives $(a, b, c: P<0,05 ; \alpha, \beta: P<0,01)$.
}

$4^{e}$ vêlage (tableau I). L'écart de poids est resté stable (de l'ordre de $20 \mathrm{~kg}$ ) entre Holstein et Montbéliardes (NS), mais a toujours été important $(P<0,01)$ entre ces 2 races et les Tarentaises $(45 \mathrm{~kg}$ entre Tarentaises et Montbéliardes). Le poids à 30 mois des $\mathrm{Hol}-$ stein et Montbéliardes a représenté $80 \%$ du poids au $4^{\mathrm{e}}$ vêlage, et $76 \%$ pour les Tarentaises $(P<0,01)$, ce qui indiquerait que les Tarentaises ont atteint moins vite leur poids adulte dans ce milieu.

Les croissances des génisses Holstein sont la première année à peine inférieures à celles réalisées par des génisses de 
même race en région de plaine océanique de l'Ouest français. Cependant, dès le second été, les croissances sont plus faibles conduisant à un poids après le premier vêlage à 3 ans inférieur de $40 \mathrm{~kg}$ environ (Troccon et al, 1993), en raison de conditions de pâturage moins favorables qu'en plaine et d'une alimentation hivernale de moins bonne qualité. Les poids à 6 mois de ces mêmes génisses correspondent à ceux enregistrés dans des troupeaux aux ÉtatsUnis (Gardner et al, 1988 ; Heinrichs et Hargrove, 1987), mais sont inférieurs dès 18 mois, et nettement inférieurs (d'environ $45 \mathrm{~kg}$ ) à 2 ans. Cela résulte sans aucun doute d'abord de niveaux alimentaires plus élevés dans les conditions américaines, correspondant à des objectifs de vêlage à 2 ans. Les poids des génisses et des vaches Montbéliardes en lactation sont semblables à ceux observés dans les départements de Franche-Comté (Herd-Book Montbéliard, 1993) pour des premiers vêlages à 33 mois. En race Tarentaise, les poids à 24 mois des génisses de Marcenat sont inférieurs de $25 \mathrm{~kg}$ à ceux enregistrés dans 15 troupeaux de Savoie, mais similaires à ceux des génisses présentées aux concours de la race (Valleix et Levast, 1987). Nos vaches en lactation ont cependant des poids supérieurs d'environ $50 \mathrm{~kg}$ à ceux relevés par Valleix et Levast (1987). Cet écart de développement à l'âge adulte pourrait s'expliquer par des conditions de milieu plus difficiles dans les Alpes, notamment en alpage. Ainsi, dans un essai conduit en alpage, la reprise de poids estivale de vaches en lactation a été 2 fois plus faible que celle observée dans notre étude (Hauwy et al, 1993 ; Coulon et D'Hour, 1994).

\section{Courbes de croissance ajustées}

Sur les 48 génisses puis vaches pesées de 5 à 56 mois, l'ajustement des courbes de croissance a permis de préciser les variations des paramètres de développement pondéral entre les races (tableau II et fig 1 ). Le poids moyen à 30 mois des 24 génisses éliminées était similaire à celui des 48 retenues pour l'analyse. Les animaux Holstein et Montbéliards ont eu les mêmes vitesses

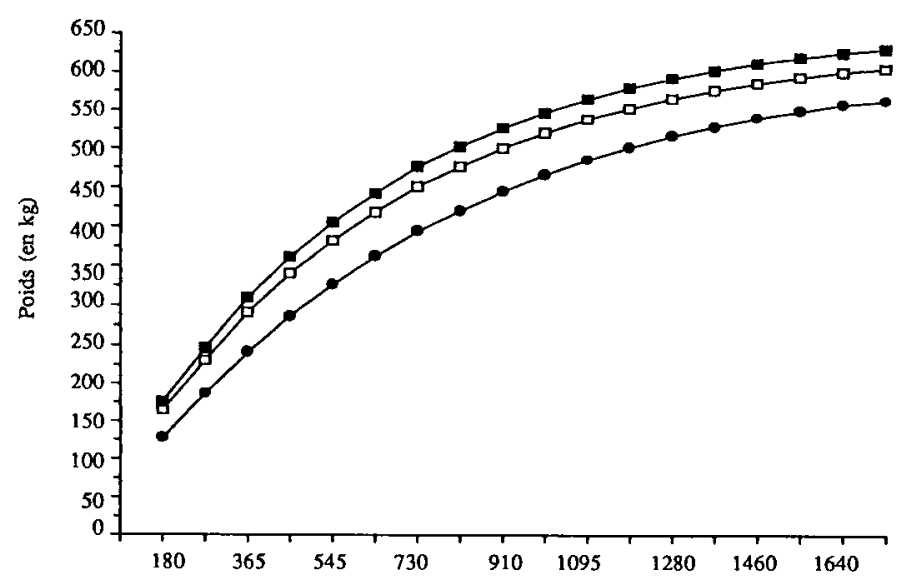

Age (jours)

Fig 1. Courbes de croissance pondérale de génisses de 3 races laitières Holstein Montbeliarde $\square$ Tarentaise ajustées selon le modèle de Brody. 
Tableau II. Moyennes des paramètres d'ajustement de la courbe de croissance de 5 à 56 mois pour des génisses laitières de 3 races par un modèle non linéaire de la forme : Poids $=P a-B \exp$ $(-k t)$, $t$ étant l'âge $(j)$.

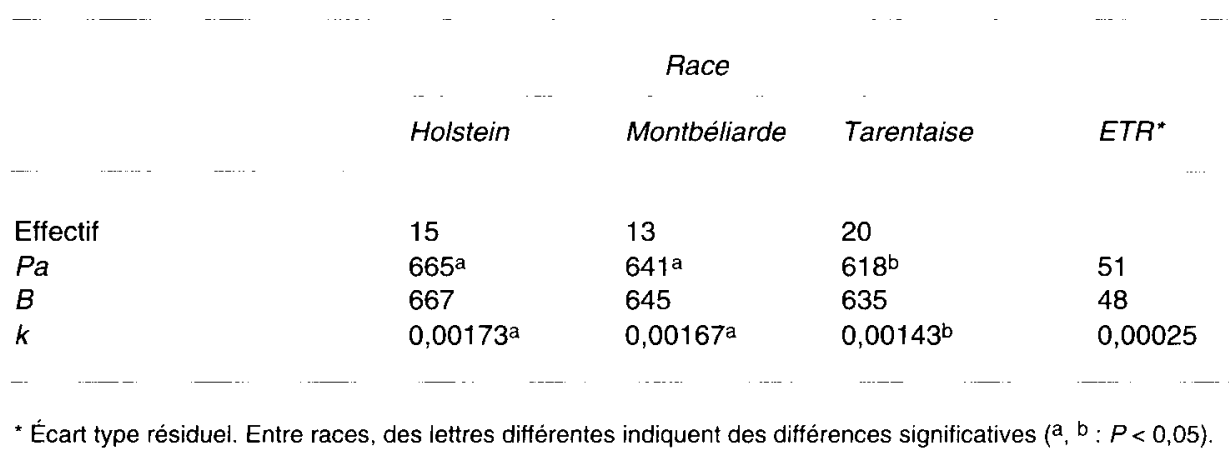

de développement pondéral $(k=0,00173$ et 0,00167 , respectivement) et leurs poids adultes estimés ne sont pas significativement différents $(\mathrm{Pa}=665$ et $641 \mathrm{~kg}$, respectivement). Le développement pondéral des génisses Tarentaises a été plus lent $(k=0,00143 ; P<0,05)$ et leur poids adulte estimé est significativement plus faible $(\mathrm{Pa}=618 \mathrm{~kg} ; P<0,05)$. À l'âge moyen de 56 mois (dernières mesures prises en compte), les vaches Holstein, Montbéliardes et Tarentaises auraient, selon ce modèle, atteint respectivement $94,8,94,3$ et $91,9 \%$ de leur poids adulte. Sous réserve que les animaux utilisés soient représentatifs de la race, le modèle met en évidence, avec un effectif réduit, les écarts relatifs de développement entre Holstein et Montbéliarde d'une part, et Tarentaise d'autre part. Ces dernières ont un poids adulte plus faible qu'elles atteindraient plus tardivement (fig 1). Cependant le poids asymptotique fourni par ce modèle surestime le poids adulte (Perotto et al, 1992). De même, l'estimée B du gain de poids total réalisé se trouve être supérieure à Pa (Villaréal, 1985). D'autre part, la croissance des Holstein du premier vêlage à 56 mois et, à un moindre degré, celle des Montbéliardes a pu être limitée dans ce milieu par le niveau d'alimentation modéré (à l'échelle de l'année) au regard de leur aptitude laitière prononcée. En effet, les Holstein ont davantage perdu de poids que les Tarentaises pendant la période hivernale de la lactation, qu'elles n'ont pas toujours rattrapé en totalité au pâturage (Coulon et D'Hour, 1994). Les conditions difficiles de milieu de cette étude, en limitant le développement à l'âge adulte des animaux les plus laitiers, auraient pu accentuer les différences entre races de vitesse de maturité jugée par la vitesse d'obtention du poids adulte. D'autres études, réalisées dans des milieux nutritionnels variés, permettraient de compléter ces observations.

\section{Développement squelettique}

Pour toutes les mensurations squelettiques réalisées, et pratiquement quel que soit l'âge, les 3 races se sont classées comme pour les poids vifs dans l'ordre décroissant suivant : Holstein, Montbéliarde et Tarentaise (fig 2), les différences entre races étant le plus souvent significatives. Cependant, pour la plupart des mensurations, l'écart entre Holstein et Montbéliardes a été faible, voire nul à 5 mois et a pris toute son impor- 
tance à un an (fig 2); cela est à rapprocher de la plus faible croissance pondérale des Montbéliardes de la naissance à 12 mois, et de leur poids plus élevé à la naissance (tableau I). En fin de période d'élevage à 35 mois, la hauteur au garrot a été significativement différente entre races $(P<0,01)$, la différence étant faible entre les génisses Holstein et Montbéliardes, les génisses Tarentaises étant nettement plus petites (tableau III). II est remarquable que la longueur du corps soit, à cet âge, semblable pour les 3 races; elle est en revanche très

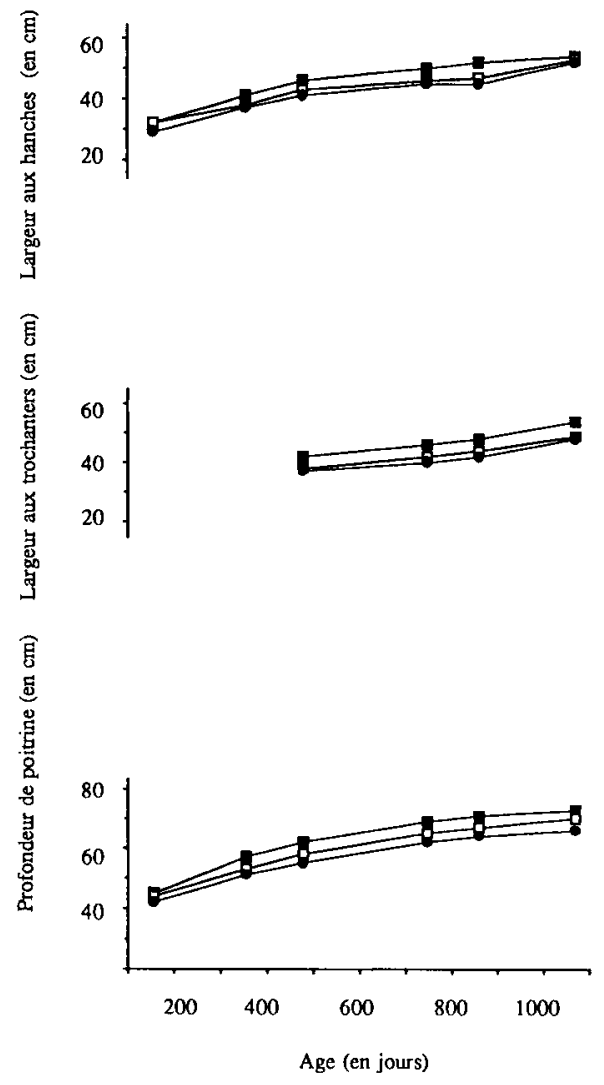

variable entre individus, ce qui pourrait résulter en partie de la difficulté de sa mesure. La cage thoracique (tour et profondeur de poitrine) était plus développée $(P<0,01)$ chez les Holstein que chez les Montbéliardes et les Tarentaises. Au niveau du bassin il y a eu peu de différences entre génisses Holstein et Montbéliardes pour la largeur aux hanches, mais davantage $(P<0,01)$ pour la largeur aux trochanters. Pour une même hauteur au garrot, les autres dimensions squelettiques (ajustées par covariance) sont restées significativement différentes entre
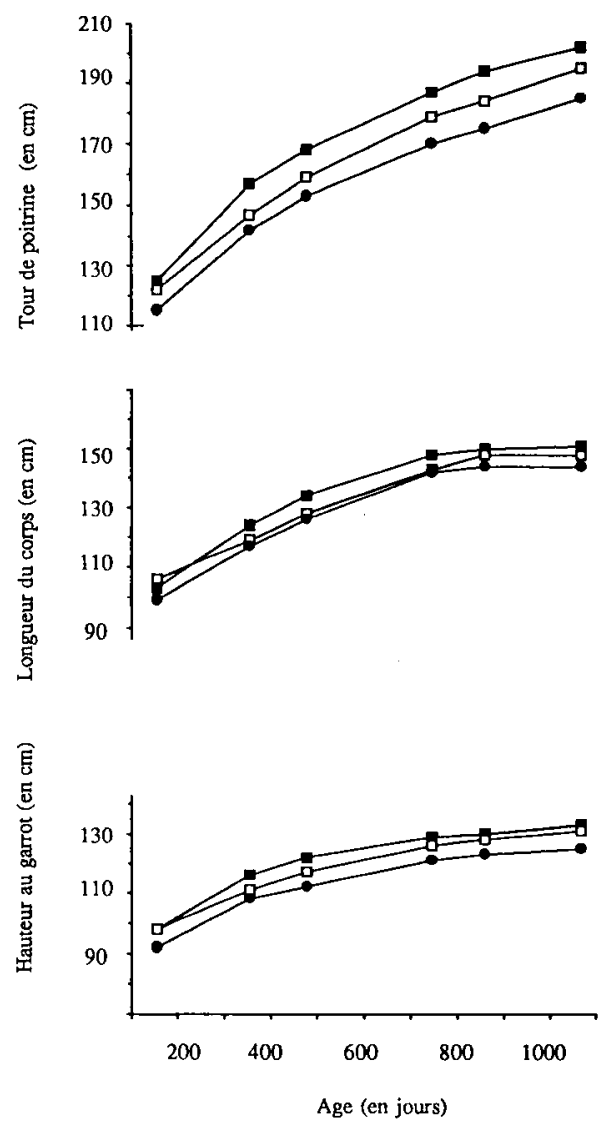

Holstein $\square$ Montbéliarde $\square \quad$ Tarentaise

Fig 2. Évolution avec l'âge des mensurations de génisses de 3 races laitières. 
Tableau III. Moyennes ajustées (moindres carrés) de paramètres de taille de génisses laitières, âgées en moyenne de 35 mois, selon leur race et leur âge.

\begin{tabular}{|c|c|}
\hline & Holstein \\
\hline- & \\
\hline Effectif & 22 \\
\hline Hauteur au garrot $(\mathrm{cm})$ & $133^{\alpha}$ \\
\hline Longueur du corps $(\mathrm{cm})$ & 145 \\
\hline Profondeur de poitrine $(\mathrm{cm})$ & $73^{a}$ \\
\hline Tour de poitrine $(\mathrm{cm})$ & $202^{a}$ \\
\hline Largeur aux hanches $(\mathrm{cm})$ & $54^{\alpha}$ \\
\hline Largeur aux trochanters $(\mathrm{cm})$ & $54^{\alpha}$ \\
\hline$\ldots$ & \\
\hline \multicolumn{2}{|c|}{$\begin{array}{l}\text { * Écart type résiduel. Entre races, des lettres différentes } \\
\text { a. } \beta, \gamma: P<0,01) \text {. }\end{array}$} \\
\hline \multicolumn{2}{|c|}{$\begin{array}{l}\text { races, excepté la largeur aux hanches. Par } \\
\text { rapport à la hauteur, les dimensions thora- } \\
\text { ciques ont été les plus élevées chez les Hol- } \\
\text { stein et les plus faibles chez les Tarentaises. } \\
\text { Il est probable que ceci soit en relation avec } \\
\text { les aptitudes laitières. }\end{array}$} \\
\hline
\end{tabular}

\section{Âge et poids à la puberté}

Les génisses Holstein ont été pubères significativement plus jeunes $(P<0,01)$ que les génisses Tarentaises et Montbéliardes (respectivement 305 j contre 475 et 504 j,
Race

Montbéliarde Tarentaise ETR*

$\begin{array}{lll}17 & 33 & \\ 131^{\beta} & 125^{\gamma} & 4 \\ 145 & 144 & 8 \\ 70^{\mathrm{b}} & 66^{c} & 2 \\ 195^{\mathrm{b}} & 185^{\mathrm{c}} & 5 \\ 53^{\alpha \beta} & 52^{\beta} & 3 \\ 49^{\beta} & 48^{\gamma} & 2\end{array}$

Tableau IV. Âge et poids moyen à la puberté de génisses laitières selon leur race.

tableau IV). L'écart type de l'âge à la puberté a été semblable dans les 3 races $( \pm 100 \mathrm{j}$ ). $90 \%$ des génisses Holstein étaient pubères dès le mois de septembre. Il a fallu attendre le mois de juin de l'année suivante pour obtenir la même proportion de génisses Montbéliardes et Tarentaises cyclées (fig 3). Le poids moyen à la puberté a été le plus faible pour les Holstein $(P<0,01)$, et comparable à ceux rapportés dans la bibliographie (Troccon et Petit, 1989). Ce sont les Montbéliardes qui ont été les plus lourdes à la puberté. Les animaux de races rustiques ou provenant de rameaux pie-rouge conti-

Race

Holstein Montbéliarde Tarentaise ETR*

$\begin{array}{lllll}\text { Effectif } & 15 & 15 & 30 & \\ \text { Âge }(\mathrm{j}) & 305^{\alpha \chi} & 504^{\beta} & 475^{\beta} & 99 \\ \text { Poids }(\mathrm{kg}) & 257^{\alpha} & 340^{\beta} & 284^{\alpha} & 44\end{array}$

${ }^{*}$ Écart type résiduel. Entre races, des lettres différentes indiquent des différences significatives $\left({ }^{(\alpha, \beta}: P<0,01\right)$. 


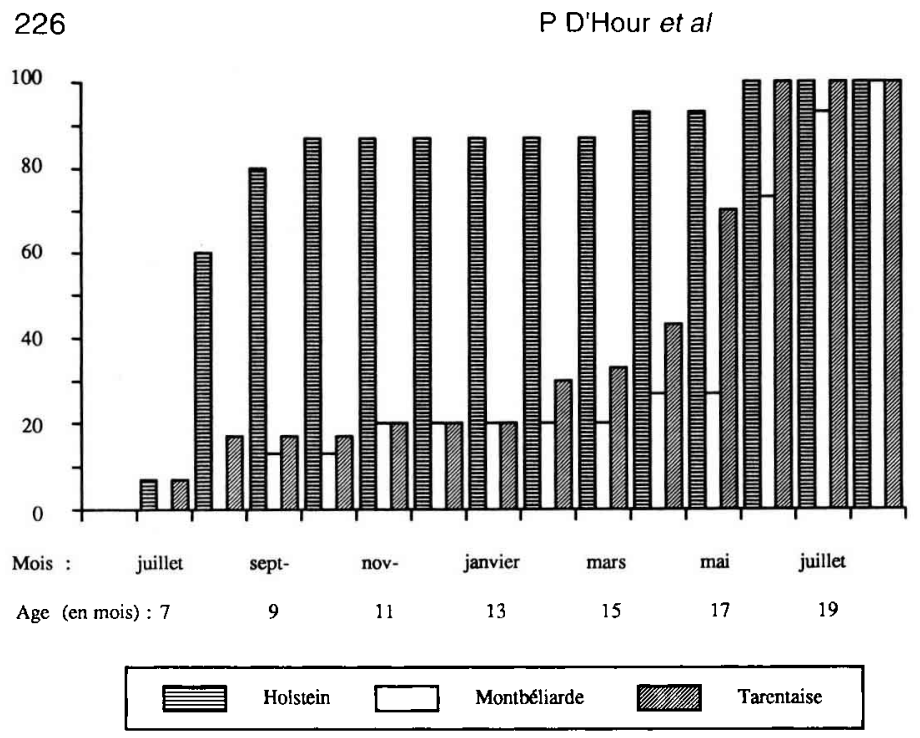

Fig 3. Évolution en fonction du mois de l'année et de l'âge de la proportion de génisses pubères de 3 races laitières.

nental sont, en règle générale, pubères plus tardivement (Laster et al, 1979; Troccon et Petit, 1989 ; Martin et al, 1992). Dans cette étude, la puberté des génisses Montbéliardes et Tarentaises a peut-être été retardée par la réduction importante de la durée du jour vers l'âge de 1 an (Hansen, 1985 ; Gauthier et al, 1986) et les faibles croissances en fin de pâturage. En effet, peu de génisses étaient cyclées en septembreoctobre vers 10 mois, leur nombre n'a pas varié en hiver et n'a commencé à augmenter qu'en avril, 1 mois avant la mise à l'herbe (fig 3).

Pour les Holstein et les Tarentaises les différences de précocité de développement et de précocité sexuelle ont été concordantes. Cela n'a pas été totalement le cas pour les Montbéliardes de cette étude, qui étaient, comme les Tarentaises, peu précoces sexuellement et ont eu pourtant un développement pondéral presque aussi précoce que celui des Holstein.

\section{CONCLUSION}

Dans des conditions particulières (nombre d'animaux restreint et d'origines peu variées ayant vraisemblablement limité leur diversité génétique, élevage d'une seule série de génisses), cette étude a permis de caractériser le développement dans un milieu montagnard de génisses Montbéliardes et Tarentaises pour lesquelles on disposait de peu de références, et de le comparer à celui mieux connu d'animaux Holstein. Les 3 races se sont classées dans l'ordre décroissant suivant pour le poids et le format: Holstein, Montbéliarde et Tarentaise. Les différences de poids et de format ont été faibles entre Holstein et Montbéliardes, la croissance des Montbéliardes étant cependant légèrement plus faible. Les Tarentaises, bien que d'un format plus réduit, ont eu un rythme d'obtention du poids adulte plus lent, en partie peut-être parce que les conditions de milieu leur ont été favorables du fait de leur plus faible niveau de production. Le développement des femelles Holstein, plus fortes productrices, a pu être ralenti en période productive. Les génisses Holstein, les plus précoces pour le développement, l'ont aussi été sur le plan de l'activité reproductrice. Les génisses Montbéliardes et Tarentaises sont pubères plus tardivement ; elles ont donc moins de possibilités de vêler vers 2 ans si les niveaux alimentaires ne sont pas nettement plus élevés 
que dans cette étude. Pour ces génisses élevées en régions montagneuses et nées au début de l'hiver, les croissances de la naissance à 18 mois sont en règle générale modérées, obligeant à un premier vêlage à un âge compris entre 33 et 36 mois.

\section{REMERCIEMENTS}

Nous remercions E Albaret, A Mante, $P$ Maronne et leur équipe du domaine INRA de Marcenat pour la réalisation des mesures et les soins aux animaux.

\section{RÉFÉRENCES}

Brody S (1945) Time relations of growth of individuals an population. In : Bioenergetics and growth (S Brody, ed), Reinhold Publ Co, reprinted 1964 by Hafner Publishing Co, New York, États-Unis, 484-574

Coulon JB, D'Hour P (1994) Effet du niveau des apports énergétiques sur les performances de vaches laitières de race Holstein ou Tarentaises. Ann Zootech $43,355-368$

D'Hour P, Coulon JB, Garel JP (1991) Capacité d'ingestion des génisses : influence de la race, de l'àge et de la qualité du foin. Ann Zootech 40, 17†-179

Fitzugh HA Jr (1976) Analysis of growth curves and strategies for altering their shape. J Anim Sci 42, 10361051

Foldager J, Sejrsen K, Larsen JB (1978) Feed intake and growth as well as the milk production in the first lactation in heifers fed ad libitum with barley, food sugar beets and long barley straw. J Dairy Sci 61 (suppl 1), 173 (abst)

Gardner RW, Smith LW, Park RL (1988) Feeding and management of dairy heifers for optimal lifetime productivity. J Dairy Sci 71, 996-999

Gauthier D, Nerot F, Garel JP, Petit M (1986) Étude de la puberté chez la génisse Salers. Influence de certains paramètres de l'environnement. INRA Bull Tech CRZV Theix 64, 55-58
Hansen ER (1985) Seasonal modulation of puberty and the post-partum anoestrus in cattle: a review. Livest Prod Sci 12, 309-327

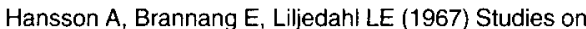
monozygous cattle twins. XIX. The interaction of heredity and intensity of rearing with regard to growth and milk yield in dairy cattle. Lantbr Högsk Ann 33 . 643-649

Hauwuy A, Bornard A, Coulon JB, Haltel L (1993) Performance des vaches laitières en alpage: effet du niveau de la complémentation en aliment concentré. INRA Prod Anim 6, 289-295

Heinrichs AJ, Hargrove GL (1987) Standards of weight and height for Holstein heifers. J Dairy Sci 70,653 660

Herd-Book Montbéliard (1993) Croissance type des génisses Montbéliardes. Résultat des pesées du concours interdépartemental. Besançon, France, $4 p$

Laster DB, Smith GM, Cundiff LV (1979) Characterisation of biological types of cattle (cycle II). J Anim Sci $48,500-508$

Little W, Kay RM (1979) The effects of rapid rearing and early calving on the subsequent performance of dairy heifers. Anim Prod 29, 131-142

Martin LC, Brinks JS, Bourdon RM, Cundiff LV (1992) Genetic effects on beef heifer puberty and subsequent reproduction. J Anim Sci 70, 4006-4017

Perotto D, Cue RI, Lee AJ (1992) Comparison of non linear functions for describing the growth of 3 genotypes of dairy cattle. Can J Anim Sci 72, 773-782

SAS (1987) SAS STAT User's Guide, Release 6.03 Edition, Cary, NC, États-Unis, 1028 p

Thimonier J (1978) L'activité ovarienne chez les Bovins. Moyens d'étude et facteurs de variations. Ann Méd Vét $122,81-92$

Troccon JL, Petit M (1989) Croissance des génisses de renouvellement et performances ultérieures. INRA Prod Anim 2, 55-64

Troccon JL, Daburon E, Gallard Y, Muller A (1993) Système d'élevage des génisses laitières. Comparaison des races Holstein et Normande. INRA Prod Anim 6, 277-288

Valleix $Y$, Levast $G$ (1987) Le format de la race Tarentaise. Étude 213. INSERM-CEIMAGREF, Grenoble France, $45 \mathrm{p}$

Villaréal EL (1985) Étude comparée des paramètres de la courbe de croissance de 4 races bovines à viande appartenant à des types génétiques différents. Thèse doct univ, Paris-Sud ( $N^{\circ} 298$ ), $119 p$ 